\title{
Discovery of a macrocyclic $\gamma$-AApeptide binding to IncRNA GAS5 and its therapeutic implication in Type 2 diabetes
}

\author{
Yan Shi ${ }^{1}$, Niketa A Patel ${ }^{2,3}$ \& Jianfeng Cai*,1 \\ ${ }^{1}$ Department of Chemistry, University of South Florida, 4202 E. Fowler Avenue, Tampa, FL 33620, USA \\ ${ }^{2}$ Department of Molecular Medicine, University of South Florida, 12901 Bruce B. Downs Boulevard, Tampa, FL 33612, USA \\ ${ }^{3}$ James A. Haley Veterans Hospital, 13000 Bruce B. Downs Boulevard, Tampa, FL 33612, USA \\ *Author for correspondence: jianfengcai@usf.edu
}

"Meanwhile, the discovery of the NP-C86 further verified the importance of the proper GAS5 level in glucose uptake and metabolism"

First draft submitted: 10 May 2019; Accepted for publication: 17 June 2019; Published online: 4 October 2019

Keywords: GAS5 • insulin $\bullet$ long noncoding RNAs • macrocyclic $\gamma$-AApeptide $\bullet$ Type 2 diabetes mellitus

\section{Relationship between Type 2 diabetes mellitus \& IncRNA GAS5}

Type 2 diabetes mellitus (T2DM) is one of the most common chronic diseases with features including insulin resistance and impaired insulin signal transduction. Significant efforts have been devoted to studying the underlying genetics of T2DM such as gene-encoding transcripts; however, it is realized lately that the regulatory noncoding transcripts play an important role in T2DM [1].

As a new class of transcripts, long noncoding RNAs (lncRNAs) regulate important biological processes and play a critical role in normal pathological and physiological processes. Recent reports have linked the aberrant expression of regulatory IncRNAs to various human diseases [2]. Among them, the lncRNA GAS5 is believed to be involved in a number of cancers and diabetes. In fact, previous studies have revealed that the level of GAS5 is decreased in T2DM patient serum [3], but the underlying mechanism remains unknown. To understand the role of GAS5 in T2DM, we recently attempted to investigate the relationship between the GAS5 and T2DM in adipocytes tissue. In this work, a one-bead two-compound screening library was first used to identify a peptidomimetic ligand that could bind to lncRNA target and serve as a probe to elucidate the role of GAS5 in T2DM [4].

\section{Depletion of GAS5 causes the glucose uptake cut-down}

Adipose tissue (AT) is an organ that regulates glucose uptake, secretes metabolites, in addition to developing insulin resistance - thus it was chosen to study the function of GAS5 in glucose metabolism. As aforementioned, the T2DM patients have a reduced level of GAS5 in serum; interestingly, we also found that in the T2DM patients' AT, the GAS5 level was lower than nondiabetic ones. To determine the causality of the decreased GAS5 level and the T2DM, the adipocytes of nondiabetic mellitus (NDM) were incubated with high concentration glucose. After 5 days, the real-time qPCR results showed that the GAS5 level was not affected by the hyperglycemia. In contrast, when GAS5 was depleted by transfecting the small interfering RNA (siRNA) in NDM adipocytes, there was a cut-down of insulin-mediated glucose uptake, indicating the regulating effect of GAS5 in glucose uptake. Another evidence to support this finding is that the translocation pathway of the insulin-mediated glucose transporter GLUT4 was inhibited in GAS5-depleted NDM adipocytes [5]. To deeply understand the influence of the depletion of GAS5 on the glucose metabolism, the genes involved in glucose metabolism were studied. The results show that the expression of insulin receptors (IR-A and B) was inhibited.

\section{GAS5 binds to the IR promotor}

Next, the chromatin-immunoprecipitation (ChIP) assay was performed to investigate whether the GAS5 could bind to the IR promotor as a transcription initiator [4,6]. To our delight, the computational modeling suggested that 
the GAS5 binds the region that was evaluated as one of the putative-binding regions on the IR promotor, implying that the GAS5 could activate the transcription of insulin receptor. The later experiment also suggested that there was a feedback loop between the insulin level and the GAS5 expression, as the hyperinsulinemia promoted the GAS5 level.

\section{GAS5 regulates the insulin-signaling pathway}

In order to know whether the GAS5 depletion regulated the insulin-signaling pathway, the phosphorylation of AKT, a downstream kinase of the insulin-signaling pathway, was evaluated. As anticipated, after the treatment of the depleted GAS5, the phosphorylation of AKT dropped significantly, while the total AKT level remained the same. It is known that there are two main inducements of T2DM, the decrease of the glucose metabolism and the insulin resistance $[7,8]$, both of which can be modulated by GAS5. As a result, we speculated if the GAS5 could be stabilized by a molecule, then the GAS5 level would be brought back to the predisease state.

\section{Relationship between GAS5 \& UPF1}

It was revealed by previous studies that the GAS5 expression was not regulated by its transcription but the degradation [9]. There was a termination codon in GAS5 transcripts, leading to the nonsense-mediated RNA decay at the initiation of the transcription. It is found that when the level of UPF1 (an essential component of nonsensemediated RNA decay) is dropped, the level of GAS5 is constantly enhanced; therefore, the strategy disrupting UPF1/GAS5 could potentially re-establish GAS5 level. Nevertheless, UPF1 function cannot be fully inhibited due to its crucial role in gene expression. It is known that UPF1 binds to the poly(A) in the stop codon of GAS5. As a result, we wondered whether it was possible to find a small molecule bound to the same region, then the binding of UPF1 to GAS5 could be disrupted, thereby enhancing the stability of GAS5.

\section{Design \& preparation of cyclic $\gamma$-AApeptide library}

As such, combinatorial chemistry, which serves as a powerful tool for ligand screening, was applied here to identify small molecules target to the IncRNA GAS5 [10]. A cyclic $\gamma$-AApeptide library was then prepared [11]. As a new class of peptidomimetics, $\gamma$-AApeptides not only retains the function and message of $\alpha$-peptides, but also exhibits improved protease resistance, chemodiversity, cellular translocation capability and bioavailability [12]. The library was synthesized on TentaGel resin by split-and-pool method. It is no longer feasible to use MS/MS to directly sequence a cyclic compound, therefore in this library, each bead was designed to bear bilayer (one-bead-twocompound, OBTC). The outer layer was the binding ligand cyclic $\gamma$-AApeptide, while the inner layer was an $\alpha$-peptide, synthesized with the Dde (1-(4,4-dimethyl-2,6-dioxacyclohexylidene)ethyl) protected $\alpha$-amino acids, as the decoding sequence. The library had a theoretical diversity of 214,375.

The OBTC library was screened against fluorescein-tagged oligonucleotide in the presence of $\sim 1000$-fold tRNAs as competitors. The oligonucleotide target contained the UPF1-binding site poly(A) tail on the $3^{\prime}$ end of transcripts, but excluded the $5^{\prime}$ binding domain which interacted with the IR. Under the fluorescence microscope, putative hits emitting bright green light were picked up. After being denatured and thoroughly washed, these hits were cleaved and sequenced by the MS/MS, and then FITC and non-FITC-labeled cyclic $\gamma$-AApeptide sequences were resynthesized. Purified by HPLC, one of the hits NP-C86 was identified as an effective ligand bound to GAS5 with high affinity $(\mathrm{kd}=153 \mathrm{nM})$.

\section{NP-C86 from the peptide library stabilizes GAS5 levels}

To verify whether the NP-C86 could disrupt the binding of GAS5 and UPF1, RNA immunoprecipitation (RIP) assay of UPF1 was performed [4,13]. The result suggested that $20 \mathrm{nM} \mathrm{NP-C86}$ could significantly reduce the level of UPF1 in DM adipocytes. Moreover, DM adipocytes were treated with NP-C86 in a dose-dependent manner (10-200 nM), then the level of GAS5, IR-A, IR-B and Glu4 were measured. Again, even at $20 \mathrm{nM}$, the level GAS5, IR-A and IR-B could be boosted dramatically. At the same concentration, the GAS5 without affecting the UPF1 level and causing cellular toxicity. When DM adipocytes were incubated with $20 \mathrm{nM} \mathrm{NP}-\mathrm{C} 86$ for $24 \mathrm{~h}$, the glucose uptake was restored to NMD level.

\section{Conclusion}

With the assistance of a chemical probe, this study has demonstrated the role of lncRNA GAS5 in T2DM and revealed its regulation in insulin receptor expression and signaling pathway. Meanwhile, the discovery of the NP- 
C86 further verified the importance of the proper GAS5 level in glucose uptake and metabolism, and in the future, it might serve as a probe to understand the mechanisms of GAS5 in other diseases and a potential drug candidate to treat T2DM. Systematic in vivo studies on a mouse model are currently underway to further evaluate its therapeutic potential. We also hope our study could shed light on the identification of novel small molecular probes or drug candidates that target other lncRNAs.

\section{Financial \& competing interests disclosure}

The authors have no relevant affiliations or financial involvement with any organization or entity with a financial interest in or financial conflict with the subject matter or materials discussed in the manuscript. This includes employment, consultancies, honoraria, stock ownership or options, expert testimony, grants or patents received or pending, or royalties.

No writing assistance was utilized in the production of this manuscript.

\section{References}

1. Quan Z, Zheng D, Qing H. Regulatory roles of long noncoding RNAs in the central nervous system and associated neurodegenerative diseases. Front. Cell. Neurosci. 11(175), 175-188 (2017).

2. Ding L, Wang M, Sun D, Li A. TPGLDA: novel prediction of associations between lncRNAs and diseases via lncRNA-disease-gene tripartite graph. Sci. Rep. 8(1), 1065 (2018).

3. Carter G, Miladinovic B, Patel AA, Deland L, Mastorides S, Patel NA. Circulating long noncoding RNA GAS5 levels are correlated to prevalence of Type 2 diabetes mellitus. BBA Clin. 4, 102-107 (2015).

4. Shi Y, Parag S, Patel R et al. Stabilization of lncRNA GAS5 by a small molecule and its implications in diabetic adipocytes. Cell Chem. Biol. 26(3), 319.e316-330.e316 (2019).

5. Elmendorf JS. Fractionation analysis of the subcellular distribution of GLUT-4 in 3T3-L1 adipocytes. Methods Mol. Med. 83, 105-111 (2003).

6. Tang R, Zhang G, Wang Y-C, Mei X, Chen S-Y. The long noncoding RNA GAS5 regulates transforming growth factor $\beta$ (TGF- $\beta$ )-induced smooth muscle cell differentiation via RNA Smad-binding elements. J. Biol. Chem. 292(34), 14270-14278 (2017).

7. Blüher M, Michael MD, Peroni OD et al. Adipose tissue selective insulin receptor knockout protects against obesity and obesity-related glucose intolerance. Dev. Cell 3(1), 25-38 (2002).

8. Kitamura T, Kahn CR, Accili D. Insulin receptor knockout mice. Annu. Rev. Physiol. 65(1), 313-332 (2003).

9. Raho G, Barone V, Rossi D, Philipson L, Sorrentino V. The gas 5 gene shows four alternative splicing patterns without coding for a protein. Gene 256(1), 13-17 (2000).

10. Lam KS, Lebl M, Krchňák V. The one-bead-one-compound combinatorial library method. Chem. Rev. 97(2), 411-448 (1997).

11. Shi Y, Challa S, Sang P et al. One-bead-two-compound thioether bridged macrocyclic $\gamma$-AApeptide screening library against EphA2.J. Med. Chem. 60(22), 9290-9298 (2017).

12. Shi Y, Teng P, Sang P, She F, Wei L, Cai J. $\gamma$-AApeptides: design, structure, and applications. Acc. Chem. Res. 49(3), 428-441 (2016).

13. Dong L, Li G, Li Y, Zhu Z. Upregulation of long noncoding RNA GAS5 inhibits lung cancer cell proliferation and metastasis via miR-205/PTEN axis. Med. Sci. Monit. 25, 2311-2319 (2019). 
